\title{
Disruption of tonB in Bordetella bronchiseptica and Bordetella pertussis prevents utilization of ferric siderophores, haemin and haemoglobin as iron sources
}

\author{
Mary Lou Nicholson and Bernard Beall
}

Centers for Disease Control and Prevention, Respiratory Diseases Branch, 1600 Clifton Rd Mailstop C02, Atlanta, GA 30333, USA
Author for correspondence: Bernard Beall. Tel: +1 404639 1237. Fax: +1 4046393123. e-mail: beb0@cdc.gov

The Bordetella bronchiseptica tonB gene was cloned by detection of a chromosomal restriction fragment hybridizing with each of two degenerate oligonucleotides that corresponded to Pro-Glu and Pro-Lys repeats characteristic of known TonB proteins. The $\operatorname{ton}_{\mathrm{Bb}}$ gene was situated upstream of exbB and exbD homologues and downstream of a putative Fur-regulated promoter. Hybridization results indicated that the ton $B$ operon and flanking regions were highly conserved between $B$. bronchiseptica, Bordetella pertussis and Bordetella parapertussis. Disruption of tonB in B. bronchiseptica resulted in inability to grow in iron-limiting media, and inability to utilize alcaligin, enterobactin, ferrichrome, desferroxamine $B$, haemin and haemoglobin. Although it was not possible to inactivate tonB in a clinical $B$. pertussis isolate, tonB was disrupted in a laboratory $B$. pertussis strain previously selected for the ability to grow on Luria-Bertani medium. This B. pertussis tonB mutant shared a similar iron complex utilization deficient phenotype with the $B$. bronchiseptica ton $B$ mutant. The $B$. bronchiseptica ton $B$ operon present on a plasmid did not complement an Escherichia coli tonB mutant, but inefficient reconstitution of enterobactin utilization was observed in one fepA mutant harbouring plasmid copies of the $B$. pertussis fepA homologue and ton $B_{\mathrm{Bb}}$ operon.

Keywords: Bordetella, iron sources, TonB-dependent receptors

\section{INTRODUCTION}

The acquisition of iron-siderophores, haem/haemin, transferrin, vitamin $\mathrm{B}_{12}$ and certain colicins in various Gram-negative bacteria is mediated through an energycoupled process, in which outer-membrane receptors and the cytoplasmic membrane proteins TonB, ExbB and ExbD are involved (Klebba et al., 1993; Postle, 1993; Braun, 1995). It is likely that TonB-dependent iron complex uptake in mammalian hosts is of key importance for Gram-negative pathogens. For example, a Haemophilus influenzae tonB mutant proved to be

Abbreviations: DP, 2,2'-dipyridyl; EDDA, ethylenediamine-di-(o-hydroxy phenylacetic acid); LB, Luria-Bertani; SS, Stainer-Scholte minimal broth lacking added iron; SS-EDDA, SS agar containing $45 \mu \mathrm{g} \mathrm{EDDA} \mathrm{ml}^{-1}$.

The GenBank accession number for the sequence reported in this paper is AF087669. avirulent in a rat model (Jarosik et al., 1994), and a Vibrio cholerae tonB mutant failed to cause disease in infant mice (Henderson \& Payne, 1994).

In current models for TonB-dependent energy transduction, the proline-rich TonB protein is anchored to the cytoplasmic membrane by its $\mathrm{N}$-terminus while the remainder of the protein occupies the periplasmic space and makes contact with outer-membrane receptors (Klebba et al., 1993; Postle, 1993; Braun, 1995). Contact by TonB with outer-membrane receptors is believed to induce a conformational change of the receptors, resulting in transport of bound ligands into the periplasmic space (Skare et al., 1993). Since ligand translocation into the periplasm is energy dependent, TonB is believed to transduce the cytoplasmic membrane proton-motive force to the receptors.

In order to function efficiently, TonB requires the two 
auxiliary proteins, ExbB and ExbD. Most of ExbB is situated cytoplasmically, and it is predicted to span the cytoplasmic membrane three times, with a short $\mathrm{N}$ terminal region and a short turn situated in the periplasm (Kampfenkel \& Braun, 1993). Like TonB, ExbD is predicted to have a short $\mathrm{N}$-terminal region anchored in the cytoplasmic membrane, with most of the protein situated in the periplasm (Kampfenkel \& Braun, 1992). ExbB and ExbD interact with and activate TonB, and it is likely that the three proteins form a complex (Fischer et al., 1989b; Skare \& Postle, 1991; Ahmer et al., 1995; Braun et al., 1996).

The important mammalian respiratory pathogens Bordetella bronchiseptica and B. pertussis express outermembrane receptors that are regulated by iron availability and are believed to be involved in the uptake of iron complexes. One such receptor is BfeA, a FepA homologue, that has been shown to be required for utilization of the exogenous siderophore enterobactin (Beall \& Sanden, 1995b). More recently, the ironregulated outer-membrane proteins BfrA, BfrB and BfrC have been identified (Beall \& Hoenes, 1997; Beall, 1998). $\mathrm{BfrA}$ is specific to B. bronchiseptica, whereas $\mathrm{BfrB}, \mathrm{BfrC}$ and BfeA appear to be highly conserved among Bordetella species. All four Bordetella proteins belong to the family of TonB-dependent outer-membrane receptors, based on their high level of amino acid sequence identity to many other bacterial proteins belonging to this group. Also, all four genes are Furrepressed, induced under low-iron conditions, and contain consensus Fur-binding sequences in their promoter regions (Beall \& Sanden, 1995a, b; Beall \& Hoenes, 1997; Beall, 1998; Brickman \& Armstrong, 1995). The ligand specificities of BfrA, BfrB and BfrC are still unknown.

Presumably, Bordetella species also have a specific receptor for ferric complexes of the Bordetella siderophore, alcaligin (Moore et al., 1995; Brickman \& Armstrong, 1996a) and for complexes of exogenously supplied ferrichrome, desferrioxamine B and haemin (Beall \& Hoenes, 1997). It was of interest to determine if the uptake of these known iron sources for Bordetella requires TonB function and if $\mathrm{TonB}$ function is required by these organisms during iron-limited conditions. It is likely that Bordetella species require the ability to multiply during iron scarcity in order to establish infection.

\section{METHODS}

Plasmids and strains. These are described in Table 1.

Media, growth conditions and antibiotics. Luria-Bertani (LB) medium was used for growth of Escherichia coli and nonhaemolytic B. bronchiseptica strains B013N and 19385. Bordet Gengou agar with $5 \%$ sheep blood was used for $B$. bronchiseptica haemolytic strain F4178 and its derivatives. All Bordetella strains were assessed for the production of haemolysin by growth on Bordet Gengou medium. Stainer-Scholte minimal medium (von Koenig et al., 1988) lacking added iron supplement (SS) was used for growth of B. pertussis. LB agar containing $100 \mu \mathrm{M}$ DP (2,2'-dipyridyl) and SS containing $45 \mu \mathrm{g} \mathrm{DP} \mathrm{ml}^{-1}$ were used to test for growth under low iron availability in B. bronchiseptica and B. pertussis respectively. Iron-deficient medium for iron complex utilization bioassays was SS agar containing $45 \mu \mathrm{g}$ EDDA [ethylenediamine-di $(o-$ hydroxyphenylacetic acid] $\mathrm{ml}^{-1}$ (SS-EDDA). For iron source utilization assays, SS-EDDA was overlaid with $3 \mathrm{ml}$ soft SS agar containing $10^{6}$ cells $\mathrm{ml}^{-1}$. Disks containing $15 \mu \mathrm{l}$ of different concentrations of iron sources were laid upon the surface, and growth haloes for $B$. bronchiseptica and $B$. pertussis strains were assessed after incubation at $37^{\circ} \mathrm{C}$ for $16 \mathrm{~h}$ and $48 \mathrm{~h}$ respectively. Albomycin sensitivity was assessed in the same manner for both species, measuring zones of inhibition around albomycin-impregnated disks on SS agar overlays. LB agar and LB broth containing 60-250 $\mu \mathrm{g}$ EDDA $\mathrm{ml}^{-1}$ were used for enterobactin disk bioassays and growth experiments with E. coli tonB and fepA mutants. LB agar plates containing $200 \mu \mathrm{M}$ DP were also used for disk bioassays with E. coli mutants.

Tetracycline was used at $15 \mu \mathrm{g} \mathrm{m}^{-1}$ and ampicillin at $200 \mu \mathrm{g} \mathrm{ml}^{-1}$. Gentamicin was used at $15 \mu \mathrm{g} \mathrm{ml}^{-1}$ for Bordetella.

Iron sources. Enterobactin extracts were prepared as previously described (Porra et al., 1972; Beall \& Sanden, 1995b) and bioassay stocks were $50 \mu \mathrm{M}$. The source of alcaligin used for this work was SS broth culture supernatant obtained from the B. bronchiseptica fur mutant B013NMn 4 (Brickman \& Armstrong, 1995). The following were obtained from Sigma and the indicated stock concentrations were used in bioassays: haemin $(100 \mu \mathrm{M})$, ferrichrome (from Ustilago sphaerogena, $50 \mu \mathrm{M})$, desferrioxamine B [deferoxamine mesylate (desferal), $50 \mu \mathrm{M}]$ and human haemoglobin $\left(500 \mu \mathrm{g} \mathrm{ml}^{-1}\right)$.

Preparation of DNA. Total genomic DNA was extracted from Bordetella avium, B. bronchiseptica, B. parapertussis and B. pertussis as previously described (Beall \& Sanden, 1995a). Plasmids were prepared with Qiagen mini-prep kits.

Southern analysis. This was performed as previously described (Beall \& Sanden, 1995a). The degenerate oligonucleotides [CCNGAR]3 and [AARCCN]3, used to detect tonB, were 3 '-end-labelled with digoxigenin (NCID/CDC Biotechnology Core Facility Branch), and were used as probes for DNA hybridization at $42^{\circ} \mathrm{C}$ as described in the Genius kit (Boehringer Mannheim). Gene-specific probes were derived from pMLN1 containing the $6 \mathrm{~kb}$ chromosomal Pst I fragment from $B$. bronchiseptica. The fragments were randomly labelled with digoxigenin (Genius kit) and used as probes for highstringency DNA hybridization at $68^{\circ} \mathrm{C}$, as described by the manufacturer.

DNA sequencing. Plasmid pMLN1 and appropriate plasmid subclones were sequenced with ABI dye-deoxy terminator kits on an ABI 377 sequencer as described by the manufacturer (Applied Biosystems) using oligonucleotides annealing to the chromosomal inserts or the pUC19 multiple cloning site. The GCG Sequence Analysis Software Package (Wisconsin Package Version 9.0, Genetics Computer Group, Madison, WI, USA) was used to analyse the DNA sequence.

Transformation and conjugation. Plasmids were transformed into E. coli by standard methods. Chromosomal integration of plasmids into B. pertussis and B. bronchiseptica was facilitated by conjugation with $E$. coli donor strain SM10 as previously described (Stibitz, 1994; Beall \& Hoenes, 1997), except that counter-selection employed colicins B and Ia (Brickman \& Armstrong, 1996b). 
Table 1. Bacterial strains and plasmids

\begin{tabular}{|c|c|c|}
\hline Strain or plasmid & Genotype or characteristics* & Source or reference \\
\hline \multicolumn{3}{|l|}{ E. coli } \\
\hline SM10 & RP4-2 Tc:: Mu conjugation donor strain & Stibitz (1994) \\
\hline DH10B & $\begin{array}{l}m c r A \Delta m c r B C \Delta h s d R \Delta h s d M \text { deoR recA1 end } 11 \\
\text { lacZ } \Delta \mathrm{M} 15\end{array}$ & Gibco-BRL \\
\hline H306 & $\operatorname{ton} B$ & K. Hantke, Tübingen University \\
\hline MT912 & $\begin{array}{l}\text { thi trp purE proC leuB lacY mtl xyl rpsL azi fhuA } \\
\text { tsx supA fepA } \mathrm{Kan}^{\mathrm{R}}\end{array}$ & $\begin{array}{l}\text { M. McIntosh, E. Carolina } \\
\text { University School of Medicine }\end{array}$ \\
\hline H5058 & ts $x$ aroB cir fiu fepA & K. Hantke \\
\hline \multicolumn{3}{|l|}{ B. bronchiseptica } \\
\hline 19385 & ATCC $19385 \mathrm{Nal}^{\mathrm{R}}$ (dog isolate), nonhaemolytic & Beall \& Hoenes (1997) \\
\hline $\mathrm{B} 013 \mathrm{~N}$ & Pig isolate, nonhaemolytic & Brickman \& Armstrong (1995) \\
\hline $\mathrm{B} 013 \mathrm{NMn}^{\mathrm{R}} 4$ & $\mathrm{~B} 013 \mathrm{~N}$ fur & Brickman \& Amstrong (1995) \\
\hline F4178 & Human throat isolate, $\beta$-haemolytic & CDC collection \\
\hline \multicolumn{3}{|l|}{ B. pertussis } \\
\hline 82 & Wild-type clinical isolate, $\beta$-haemolytic & Beall \& Sanden (1995b) \\
\hline UT25D & Derivative of UT25, nonhaemolytic & Brown \& Parker (1987) \\
\hline \multicolumn{3}{|l|}{ B. parapertussis } \\
\hline A168 & Wild-type clinical isolate & Beall \& Sanden (1995b) \\
\hline \multicolumn{3}{|l|}{ B. avium } \\
\hline BA10 & Wild-type & Beall \& Sanden (1995b) \\
\hline \multicolumn{3}{|l|}{ Plasmids } \\
\hline pSS2141 & Broad-host-range integrational vector, $\mathrm{Gm}^{\mathrm{R}} \mathrm{Ap}^{\mathrm{R}}$ & Stibitz (1994) \\
\hline pRK415 & Broad-host-range replicative vector, Tet $^{\mathrm{R}}$ & Keen et al. (1988) \\
\hline pRKTon & $\begin{array}{l}\text { pRK415 derivative containing } B . \text { bronchiseptica } \\
\text { tonB-exbB-exbD operon }\end{array}$ & This work \\
\hline pSSt2, pSSt3 & $\begin{array}{l}\text { pSS2141 containing tonB gene fragment for } \\
\text { integrational inactivation of } \operatorname{ton} B_{\mathrm{Bb}}\end{array}$ & This work \\
\hline pSSt1 & pSS2141 containing $5^{\prime}$ end of $\operatorname{ton} B_{\mathrm{Bb}}$ gene & This work \\
\hline pKp1 & PUC19 derivative containing $b f e A$ and promoter & Beall \& Sanden (1995b) \\
\hline
\end{tabular}

$* \mathrm{Ap}^{\mathrm{R}}, \mathrm{Gm}^{\mathrm{R}}, \mathrm{Kan}^{\mathrm{R}}, \mathrm{Nal}^{\mathrm{R}}, \mathrm{Tet}^{\mathrm{R}}$, denote resistance to ampicillin, gentamicin, kanamycin, nalidixic acid and tetracycline respectively.

\section{RESULTS}

\section{Sequence of the $B$. bronchiseptica ton $B-e x b B-e x b D$ genes}

In order to clone the ton $B$ gene from $B$. bronchiseptica, we designed two degenerate oligonucleotide probes, $[\mathrm{CCNGAR}] 3$ and [AARCCN]3, corresponding to prominent (Glu-Pro) ${ }_{n}$ and (Lys-Pro) ${ }_{n}$ repeats characteristic of many TonB proteins. This strategy relied upon identifying identically sized genomic restriction fragments that hybridized in independent Southern blots with each of the two degenerate primers. In this way, a $6 \mathrm{~kb}$ Pst I fragment was identified (Fig. 1), and subsequently cloned into pUC19 to generate pMLN1.

The complete nucleotide sequence of the $2.4 \mathrm{~kb} S m a \mathrm{I}$ DNA fragment situated within the $6 \mathrm{~kb}$ Pst I fragment was determined. An examination of the sequence revealed three open reading frames directed in the same orientation. Because of obvious homology with characterized proteins, these open reading frames were sequentially designated $t o n B_{\mathrm{Bb}}, e x b B_{\mathrm{Bb}}$ and $e x b D_{\mathrm{Bb}}$. As in Neisseria species, Haemophilus influenzae, Pseudomonas putida and Xanthomonas campestris, these genes appear to be organized in an operon in B. bronchiseptica
(Bitter et al., 1993; Jarosik et al., 1994; Stojiljkovic \& Srinivasan, 1997; Wiggerich et al., 1997). The exbB and $e x b D_{\mathrm{Bb}}$ genes are predicted to have efficient ribosome-binding sites compared to the ribosomebinding site for $\operatorname{ton} B_{\mathrm{Bb}}$. This feature is likely to be essential in production of sufficient levels of these proteins for efficient TonB activation.

The 268-codon $\operatorname{ton} B_{\mathrm{Bb}}$ showed strong homology to various Gram-negative TonB proteins, with 30-39\% identity between overlaps of about 200-270 residues. These included the TonB proteins from E. coli, Salmonella typhimurium, Klebsiella pneumoniae, Enterobacter aerogenes, Serratia marcescens, Yersinia enterocolitica, Pseudomonas aeruginosa, P. putida, X. campestris, H. influenzae and Neisseria meningitidis (GenBank accession numbers K00431, X56434, X68478, A36928, X60996, Q05741, U23764, S28444, Z95386, U04996 and U77738 respectively). A feature of $\mathrm{TonB}_{\mathrm{Bb}}$ shared with other TonB proteins includes a short hydrophobic $\mathrm{N}$-terminal putative transmembrane anchor (residues 20-40) with the remainder constituting a mainly hydrophilic putative periplasmic domain. Like other TonB proteins, the B. bronchiseptica TonB is proline-rich $(21 \%)$ and exhibits Lys-Pro and Glu-Pro 
(a)

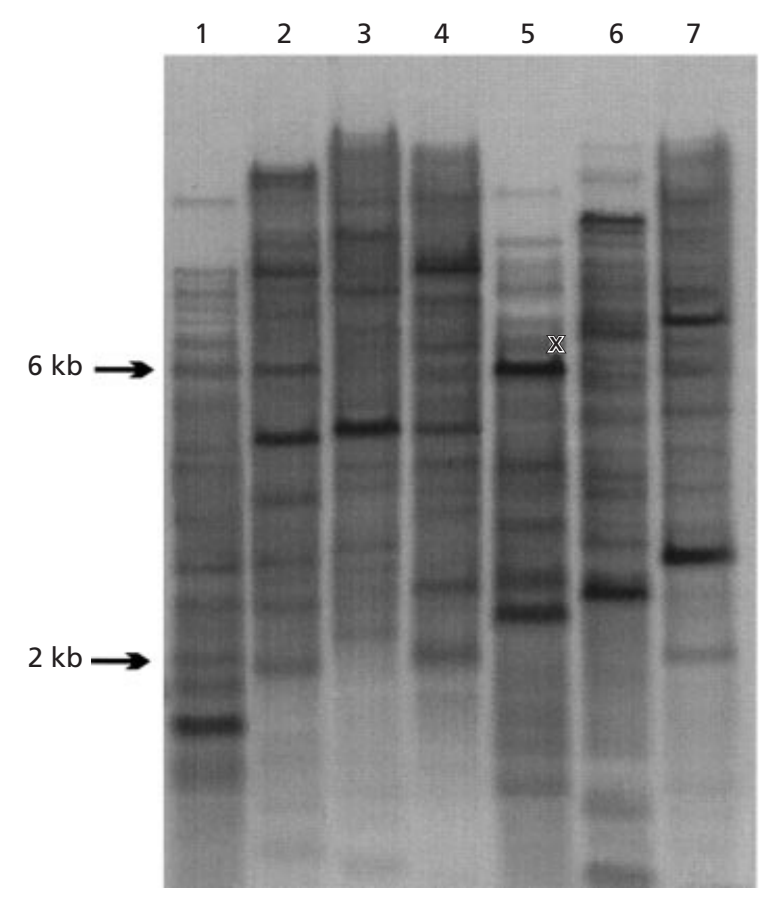

(b)

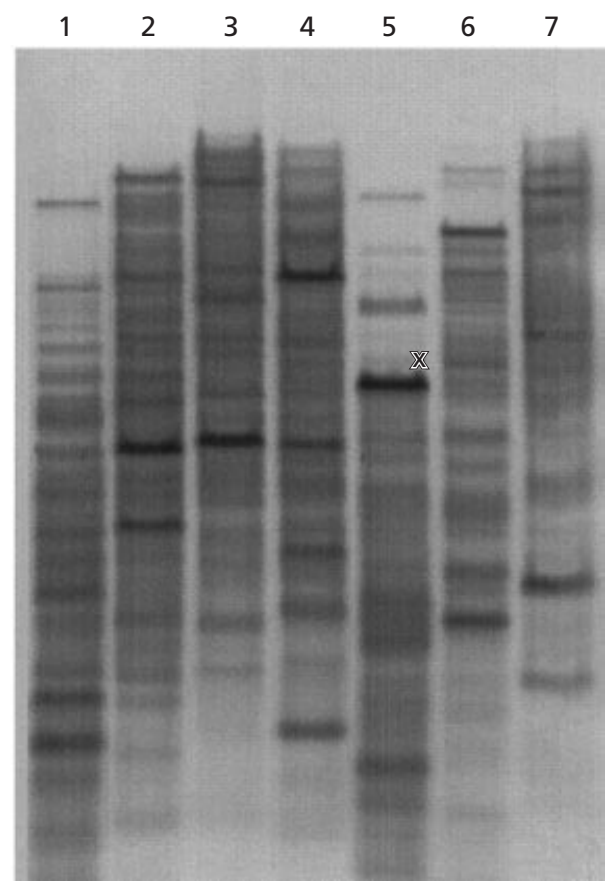

Fig. 1. Southern blot of $B$. bronchiseptica DNA digests using degenerate oligonucleotides [CCNGAR]3 (a) and $[A A R C C N] 3$ (b) as digoxigenin-labelled probes. DNA was digested with Accl (lane 1), Aatll (lane 2), BamHI (lane 3), EcoRI (lane 4), Pstl (lane 5), Smal (lane 6) and Xhol (lane 7). The $6 \mathrm{~kb}$ Pstl fragment containing the tonB-exbB-exbD operon is indicated by an $\mathrm{x}$.

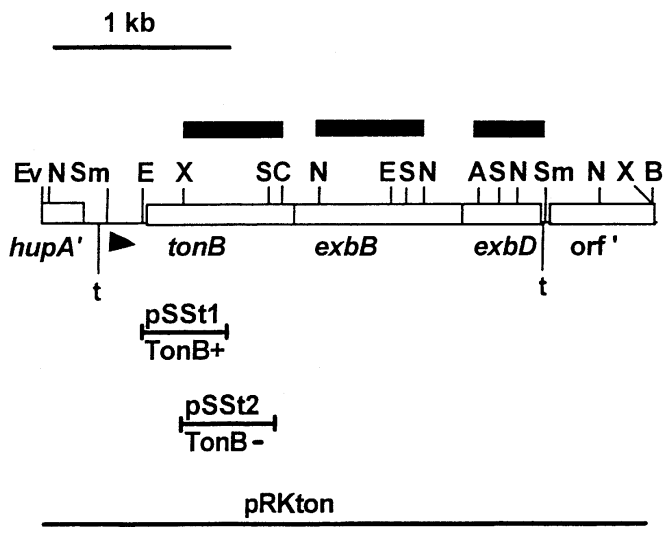

Fig. 2. Diagram of the ton $B-e x b B-e x b D$ region of $B$. bronchiseptica. A putative promoter and direction of transcription is indicated by an arrow. Two potential terminators are labelled ' $\mathrm{t}$ '. hupA' and orf' represent two truncated open reading frames described in the text. Black rectangles represent gene-specific probes used for the Southern analysis shown in Fig. 3. pSSt1 and pSSt2 indicate Campbelltype insertions of the indicated plasmids into Bordetella strains with the iron-utilization phenotype $\left(\mathrm{TonB}^{+}\right.$or $\left.\mathrm{TonB}^{-}\right)$shown. The entire EcoRV-BamHI fragment present in the replicative plasmid pRKton used for complementation studies is represented. Restriction enzymes: A, Aatll; B, BamHI; C, Clal; E, EcoRI; N, Ncol; S, Sall; Sm, Smal; X, Xhol.

repeats (7 and 12 repeats respectively) typical of previously described TonB proteins.

Located $1 \mathrm{bp}$ downstream of the tonB stop codon was the 314-codon $e x b B_{\mathrm{Bb}}$ gene, which has deduced homology to various ExbB proteins (about $29-41 \%$ sequence identity over $85-286$ residues). The closest matching ExbB homologue to $\mathrm{ExbB}_{\mathrm{Bb}}$ is from X. campestris, with $41 \%$ sequence identity (GenBank accession number Z95386).

The 155-codon exbD $\mathrm{Bb}_{\mathrm{Bb}}$ gene overlapped by 1 base with the $\operatorname{exb} B_{\mathrm{Bb}}$ stop codon. The deduced $\mathrm{ExbD}_{\mathrm{Bb}}$ protein sequence shows the most similarity to the $N$. meningitidis and $X$. campestris homologues, with 36$40 \%$ identity (GenBank accession numbers U77738 and Z95386 respectively).

Directly upstream of $t o n B_{\mathrm{Bb}}$ are potential -35 and -10 hexamers homologous to the consensus sequence of $E$. coli $\sigma^{70}$-directed promoters (Hawley \& McClure, 1983). This putative promoter sequence is overlapped by a sequence similar to Fur-binding sites. The sequence from bases 86 to 105 (see accession AF087669) is identical to the consensus Fur-binding site in 12 of 19 positions (Calderwood \& Mekalanos, 1987), and was the only 'iron box' found with a homology search of the entire $2446 \mathrm{bp}$. About $160 \mathrm{bp}$ upstream of tonB are two $\mathrm{G}+\mathrm{C}$-rich inverted repeats between bases 10-39, either of which could possibly function in transcription termination. Partial sequence analysis of the open reading frame immediately upstream of the sequence shown in accession AF087669 revealed a gene highly homologous to genes encoding bacterial histone-like proteins, including S. typhimurium hupA (Higgins \& Hillyard, 1988). Eighty bases downstream of exbD and down- 
(a) $\operatorname{ton} B$

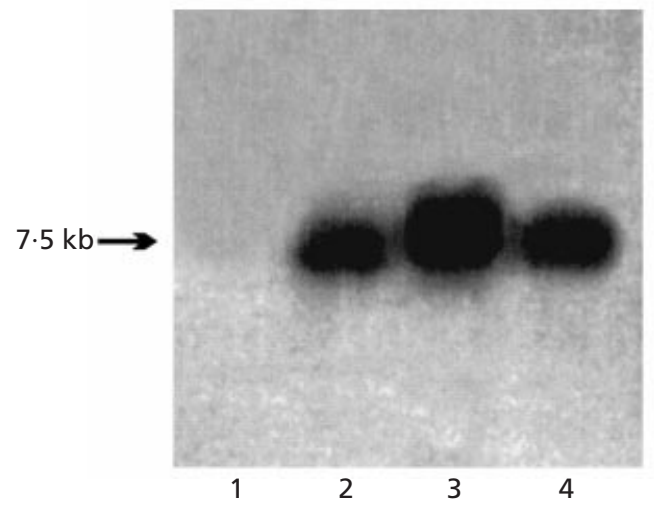

(b) exbB

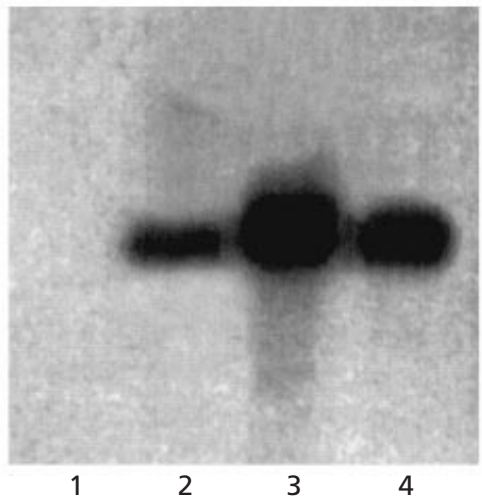

(c) exbD

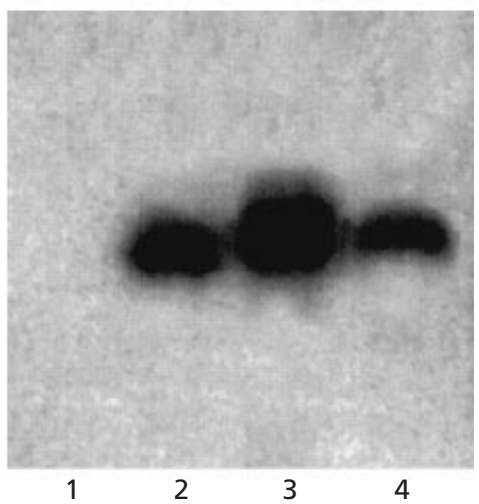

Fig. 3. Southern analysis of $B$. avium (lane 1), B. bronchiseptica (lane 2), B. parapertussis (lane 3) and B. pertussis (lane 4) digested with $A a t l l$ and probed with the gene-specific probes ton $B(a)$, exbB (b) and exbD (c).

stream of a potential transcriptional terminator was an open reading frame with high homology to various 'two-component system' DNA-binding regulatory proteins (Fig. 2).

\section{Conservation of the tonB-exbB-exbD operon in Bordetella species}

The $\operatorname{ton} B_{\mathrm{Bb}}, e x b B_{\mathrm{Bb}}, e x b D_{\mathrm{Bb}}$ genes and flanking regions appear to be highly conserved between $B$. bronchiseptica, B. pertussis and B. parapertussis, since DNA from each of these three species had apparently identically sized AatII restriction fragments that hybridized strongly to the three gene fragment probes (Fig. 3a-c, lanes 2-4). In addition, B. bronchiseptica and B. pertussis shared identically sized EcoRI and XhoI restriction fragments that hybridized to each of the gene fragment probes (data not shown). Since XhoI, EcoRI and AatII have single sites within the $\operatorname{ton} B_{\mathrm{Bb}}, e x b B_{\mathrm{Bb}}$ and $e x b D_{\mathrm{Bb}}$ genes respectively (Fig. 3), these results indicate that these three genes and their flanking regions are highly conserved between the two species. These results also show that the putative $B$. avium homologues of these genes are not detectable by high-stringency Southern analysis (Fig. 3a-c, lane 1).

\section{Construction of B. bronchiseptica and B. pertussis tonB mutants}

Two chromosomal fragments were used for plasmid integrational analysis of the tonB gene in B. bronchiseptica and in B. pertussis (Fig. 2). Plasmid pSSt1 was constructed by cloning a $294 \mathrm{bp}$ PCR fragment consisting of bases 209-502 including the first 60 codons of $\operatorname{ton}_{\mathrm{Bb}}$ and $4 \mathrm{bp}$ of upstream sequence, into the EcoRI site of pSS2141 (Stibitz, 1994) immediately downstream of the gentamicin-resistance gene such that the ton $B$ fragment was in the same orientation as the gentamicinresistance gene. Plasmids pSSt 2 and pSSt 3 consisted of the $506 \mathrm{bp}$ internal $\operatorname{ton}_{\mathrm{Bb}}$ XhoI-SalI fragment cloned into the SalI site of pSS2141 in either orientation (bases
345-851). These plasmids were subsequently integrated into the chromosome of B. bronchiseptica strains 19385 , $\mathrm{B} 013 \mathrm{~N}$ and $\mathrm{F} 4178$ by conjugation with the E. coli donor strain SM10 followed by selection for colicin and gentamicin resistance. Since pSSt 2 and pSSt 3 contained an internal fragment lacking both the $5^{\prime}$ and $3^{\prime}$ ends of the $\operatorname{ton} B_{\mathrm{Bb}}$ structural gene, simple homologous insertion of the plasmid resulting from a single crossover event within the $\operatorname{ton} B$ gene resulted in a truncated $\operatorname{ton} B_{\mathrm{Bb}}$ gene of 218 codons.

Attempts to insertionally inactivate ton $B$ in the clinical B. pertussis strain 82 with plasmids pSSt 2 and pSSt3 were unsuccessful. Transconjugants were obtained through matings of strain 82 with SM10(pSSt2) and SM10(pSSt3), but at a very low frequency. Chromosomal DNA was prepared from one transconjugant, digested with EcoRI, and then ligated. This was followed by transformation of E. coli DH10B to ampicillin resistance. Plasmid DNA from several transformants from this chromosome-walking procedure revealed that these plasmids did not contain restriction fragments of the sizes predicted for $\mathrm{pSSt} 2$ or $\mathrm{pSSt} 3$ derivatives that had integrated into the strain 82 tonB gene (data not shown). In contrast, transconjugants were readily obtained by conjugation of plasmids pSSt 2 and pSSt 3 into B. pertussis strain UT25D. This strain is a nonhaemolytic derivative of UT25 (Brickman \& Armstrong, 1995) that has been adapted to grow on LB agar. In contrast to strain 82 transconjugants, plasmid pSSt 2 and pSSt 3 integrations into UT25D and the two B. bronchiseptica wild-type strains were found to occur as predicted by a single homologous crossover event. This resulted in truncation of $50 \mathrm{C}$-terminal residues of TonB in the $B$. bronchiseptica strains and a similar if not identical truncation of TonB in B. pertussis strain UT25D.

Our stock B. bronchiseptica strains 19385 and B013N were non-haemolytic on Bordet Gengou agar, possibly due to laboratory passage resulting in phase variation or modulation (Weiss \& Hewlett, 1986), although it has 
Table 2. TonB-dependent phenotypes in wild-type and Campbell insertion derivatives of $B$. bronchiseptica and B. pertussis

\begin{tabular}{|c|c|c|c|c|c|}
\hline Strain* & $\operatorname{ton} B$ & $\mathrm{LB}-\mathrm{DP} \phi$ & SS-DP $\$$ & $\begin{array}{c}\text { Iron } \\
\text { utilization§ }\end{array}$ & Albomycin $\|$ \\
\hline 19385 & + & + & + & + & S \\
\hline $19340(19385 \Omega \mathrm{pSS} \mathrm{t} 1)$ & + & + & + & + & $\mathrm{s}$ \\
\hline 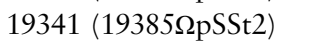 & - & - & - & - & $\mathrm{R}$ \\
\hline 19341(pRKton) & + & + & + & + & S \\
\hline 19341(pRK415) & - & - & - & - & $\mathrm{R}$ \\
\hline B013N & + & + & + & + & $\mathrm{ND}$ \\
\hline BN600 (B013N $\Omega p S S t 2)$ & - & + & + & - & $\mathrm{ND}$ \\
\hline F4178 & + & + & + & + & S \\
\hline 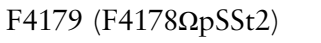 & - & - & - & - & $\mathrm{R}$ \\
\hline UT25D & + & $\mathrm{ND}$ & + & + & $\mathrm{s}$ \\
\hline BB100 (UT25D $\Omega$ pSSt2) & - & $\mathrm{ND}$ & - & - & $\mathrm{R}$ \\
\hline BB100(pRKton) & + & $\mathrm{ND}$ & + & + & S \\
\hline BB100(pRK415) & - & $\mathrm{ND}$ & - & - & $\mathrm{R}$ \\
\hline 82 & + & ND & - & + & $\mathrm{s}$ \\
\hline
\end{tabular}

ND, Not determined.

* The symbol $\Omega$ indicates that the strain contains a single homologously inserted copy of the indicated plasmid.

† Growth on LB agar containing $100 \mu \mathrm{g}$ dipyridyl $\mathrm{ml}^{-1}$.

‡Growth on SS agar containing $45 \mu$ g dipyridyl $\mathrm{ml}^{-1}$.

S Growth haloes in iron utilization assays around disks containing alcaligin, enterobactin, ferrichrome, desferrioxamine B, haemin or haemoglobin.

$\|$ Sensitivity $(S)$ indicates zones of inhibition around albomycin disks containing $10 \mu \mathrm{l}$ of a $12.5 \mu \mathrm{g} \mathrm{ml}^{-1}$ albomycin solution. Resistance $(\mathrm{R})$ indicates no zones of inhibition using $10 \mu \mathrm{l} \mathrm{of} 250 \mu \mathrm{g} \mathrm{ml}{ }^{-1}$ albomycin.

been observed that initial isolates of B. bronchiseptica from clinical specimens are often non-haemolytic. Since we were unable to insertionally inactivate ton $B$ in the clinical, haemolytic B. pertussis isolate 82 , it appeared possible that attempts to insertionally inactivate $\operatorname{ton} B$ in phase 1 haemolytic strains of $B$. bronchiseptica would be unsuccessful. However, insertional inactivation of ton $B$ in the $\beta$-haemolytic $B$. bronchiseptica human isolate F4178 was readily accomplished.

\section{Iron source utilization deficiency and albomycin sensitivity of $\operatorname{ton} B_{\mathrm{Bb}}$ mutants}

Integration of pSSt1, which contained the $5^{\prime}$ end of the structural $t o n B_{\mathrm{Bb}}$ gene, into the $B$. bronchiseptica chromosome resulted in placing the intact $\operatorname{ton}_{\mathrm{Bb}}$ gene immediately downstream of the pSS2141 gentamicinresistance gene and in the same orientation. This also resulted in replacement of the tonB ribosome-binding site with a different ribosome binding region (from ACCTGGCTGATTC-ATG to TAATTAAGAATTCATG). Campbell-type insertion of pSSt1 had no effect on the ability of B. bronchiseptica strain 19340 to grow on LB agar containing $100 \mu \mathrm{M}$ DP, nor did these transconjugants differ from the parental strain 19385 in utilization of various iron sources as measured by disk bioassays (Table 2). Therefore, the vector promoter sequence $(\mathrm{s})$ and the different $\operatorname{ton}_{\mathrm{Bb}}$ ribosome-binding site resulting from Campbell insertion of pSS2141 served to substitute for the normal $\operatorname{ton} B_{\mathrm{Bb}}$ promoter and ribosome-binding site.

In contrast, Campbell insertions of pSSt 2 or pSSt 3 resulted in the inability of $B$. bronchiseptica and $B$. pertussis UT25D transconjugants to form growth haloes in disk-bioassays around alcaligin, enterobactin, ferrichrome, desferrioxamine B and haemin (Table 2). Further indication of an alcaligin-utilization defect in transconjugant strain 19341 was its inability to grow on LB-DP and SS-DP. It was interesting to note that pSSt2 and pSSt3 Campbell integration derivatives of the dog isolate $\mathrm{B} 013 \mathrm{~N}$ were unable to utilize the various iron sources used in bioassays, but were unimpaired in their ability to grow on LB-DP and SS-DP agar. It was also interesting that the clinical B. pertussis isolate strain 82 was unable to form colonies on SS-DP agar, yet was able to utilize all of the various iron sources used in bioassays (Table 2). These observations indicate differences among strains of these species affecting growth in ironlimiting media.

Another phenotype demonstrating tonB inactivation in $B$. bronchiseptica and $B$. pertussis ton $B$ mutant strains was their marked resistance to the antibiotic albomycin (Table 2), which is structurally similar to ferrichrome. These strains showed no zones of growth inhibition around albomycin disks containing $10 \mu \mathrm{l}$ of a $250 \mu \mathrm{g}$ 
$\mathrm{ml}^{-1}$ albomycin solution, while the wild-type parental strains showed wide zones of inhibition around disks containing $10 \mu \mathrm{l}$ of $25 \mu \mathrm{g} \mathrm{ml}{ }^{-1}$ albomycin (data not shown).

\section{Complementation of $B$. bronchiseptica and $B$. pertussis ton $B$ mutants}

A $3.6 \mathrm{~kb}$ EcoRV-BamHI fragment from pMLN1 containing the intact $\operatorname{ton} B_{\mathrm{Bb}}-e x b B_{\mathrm{Bb}}-e x b D_{\mathrm{Bb}}$ operon (Fig. 2) was subcloned into the broad-host-range vector pRK415 (Keen et al., 1988), resulting in plasmid pRKton. Introduction of pRKton into the ton $B$ mutant strains $B$. bronchiseptica 19341 and B. pertussis BB100 completely restored wild-type iron-utilization and albomycin-sensitivity phenotypes (Table 2), demonstrating that the mutant phenotypes were not conferred by polarity affects on genes downstream of exbD.

\section{ton $B_{\mathrm{Bb}}$ on pRKton cannot complement an $E$. coli tonB mutant}

Plasmid pRKton could not complement the enterobactin and vitamin B12 utilization defects exhibited by the $E$. coli tonB mutant strain H306 (data not shown). The TonB-dependent receptors for colicin B, colicin Ia and albomycin were also still not functional in this mutant since H306(pRKton) was still totally resistant to each of these molecules (data not shown).

\section{ton $B_{\mathrm{Bb}}$ and $b f e A$ inefficiently reconstitute enterobactin utilization in an $E$. coli fepA mutant}

Attempts were made to reconstitute enterobactin utilization in two different E. coli fepA mutants [H5058 (fepA, aroB) and MT912 (fepA)] and in H306(tonB) harbouring the compatible plasmids pKp1 and pRKton. With H5058, H306 and their plasmid-containing derivatives, no differences were seen in disk bioassays using added enterobactin, nor were differences seen in growth assays in iron-limiting media (data not shown). However, the fepA mutant MT912(pKp1, pRKton) formed larger colonies than MT912(pKp1, pRK415) on $\mathrm{LB}$ agar containing $60 \mu \mathrm{g}$ EDDA ml ${ }^{-1}$ and this effect was measurable in LB broth containing EDDA (data not shown). These effects in liquid medium were at least partially due to a higher colony-forming efficiency of MT912(pKp1, pRKton) relative to MT912(pKp1, pRK415) in media containing 100-250 $\mu \mathrm{g}$ EDDA $\mathrm{ml}^{-1}$ $\left(10^{-4}\right.$ vs 0 colony-forming efficiency). We also found small-diameter growth haloes around enterobactin disks with MT912(pKp1, pRKton) on L agar containing $250 \mu \mathrm{g}$ EDDA ml ${ }^{-1}$ within $24 \mathrm{~h}$, whereas MT912(pKp1, pRK415) only displayed a very faint growth halo after $72 \mathrm{~h}$. This latter effect is likely to be due to Cir- and Fiumediated utilization of enterobactin degradation products (Hantke, 1990).

\section{DISCUSSION}

Although the B. bronchiseptica TonB, ExbB and ExbD proteins share only $34 \%, 29 \%$ and $32 \%$ sequence identity respectively with corresponding overlaps with their respective E. coli homologues, each of these homologue pairs have strikingly similar hydrophobicity plots, which probably reflects conserved cellular localization and function. The majority of identified TonB proteins contain Pro-Glu and Pro-Lys repeat domains in close proximity, upon which we relied for our strategy in cloning ton $B_{\mathrm{Bb}}$. The region encompassing these domains is thought to allow TonB to physically extend through the periplasm, allowing contact with outer-membrane receptors (Evans et al., 1986; Hannavy et al., 1990; Larsen et al., 1993). The histidine at residue 20 and neighbouring residues conserved among other TonB residues are believed to be important in the E. coli TonB function (Karlsson et al., 1993; Jaskula et al., 1994). There is a similar region centring at the histidine residue in $\mathrm{TonB}_{\mathrm{Bb}}$ (residue 30 ) that is situated at roughly the same position in the putative membrane-associated $\mathrm{N}$-terminus at nearly the same distance from a conserved arginine at residue 19. The PXYP motif (residues 160-163 in the E. coli TonB; $190-193$ in TonB $_{\mathrm{Bb}}$ ) thought to interact with outer-membrane TonB boxes (Heller et al., 1988; Gudmundsdottir et al., 1989; Kadner, 1990; Traub et al., 1993) is conserved among most of the TonB proteins, including $\mathrm{TonB}_{\mathrm{Bb}}$, which is consistent with the similarity of Bordetella siderophorereceptor-like protein TonB boxes to those of E. coli TonB-dependent receptors. Two proposed amphiphilic $\beta$-strands (Anton \& Heller, 1991) (encompassed between residues 173-196 for the E. coli TonB and 197-215 for $\mathrm{TonB}_{\mathrm{Bb}}$ ) with highly conserved glycine residues (positions 174 and 186 for the E. coli TonB, 203 and 215 for $\mathrm{TonB}_{\mathrm{Bb}}$ ) are conserved among all of the known TonB proteins.

As with the P. putida ExbB protein, $\operatorname{ExbB}_{\mathrm{Bb}}$ has a nonconserved N-terminal extension relative to other ExbB proteins. However, both of these ExbB proteins share highly conserved $\alpha$-helical transmembrane domains with the other known ExbB proteins. It is within these domains that ExbB is thought to interact with TonB and ExbD (Kampfenkel \& Braun, 1992; Koebnik, 1993; Larsen et al., 1994; Traub et al., 1993). These include the N-terminal region $\mathrm{VX}_{3} \mathrm{VX}_{3} \mathrm{LX}_{3} \mathrm{SX}_{3}$ motif (residues 84-102 of $\mathrm{ExbB}_{\mathrm{Bb}}$ ), a domain encompassed by residues 202-225 of $\mathrm{ExbB}_{\mathrm{Bb}}$ with four conserved glycine residues, and an alanine-rich $\alpha$-helical region between residues 240-270 with conserved asparagine and arginine residues (residues 265 and 270).

The most conserved regions between $\mathrm{ExbD}_{\mathrm{Bb}}$ and other ExbD proteins are the putative $\mathrm{N}$-terminal transmembrane region (approx. residues 17-40) and the Cterminal periplasmic region thought to interact with TonB and ExbB. The aspartate residue (residue 25 and 26 of the E. coli and B. bronchiseptica ExbD respectively), shown to be important in ExbD function (Braun et al., 1996), is conserved among known ExbD proteins; and the E. coli ExbD leucine 132, also shown to be functionally important (Braun et al., 1996), is conservatively substituted by phenylalanine in $B$. bronchiseptica (residue 133), X. campestris and N. meningitidis. It is unknown why we were unable to insertionally 
inactivate ton $B$ in a phase 1 isolate of $B$. pertussis. We have subsequently attempted ton $B$ inactivation in an independent phase 1 isolate with no success (data not shown). It is likely that this result is due to an effect of ton $B$ inactivation on processes other than iron uptake. For example, in Pseudomonas aeruginosa, tonB inactivation results in hypersensitivity to a wide variety of antibiotics in a manner that is independent of iron concentration in the growth medium (Zhao et al., 1998), although we did not note problems in selecting gentamicin resistance for the insertional inactivation of ton $B$ in the phase $1 \mathrm{~B}$. bronchiseptica strain F4178.

The $P$. aeruginosa TonB protein does not appear significantly more related to the E. coli TonB than Ton $_{\mathrm{Bb}}$, yet the $P$. aeruginosa TonB is known to function in $E$. coli ton $B$ mutants, facilitating the utilization of several different receptors (Poole et al., 1996). TonB proteins of Neisseria species show the same overall conservation of key sequence features with the E. coli TonB; however, the N. meningitidis TonB apparently cannot complement $E$. coli in the utilization of its TonB-dependent receptors (Stojiljkovic \& Srinivasan, 1997). Reconstitution of the N. meningitidis haemoglobin-utilization system in E. coli was successful, employing expression of the $N$. meningitidis haemoglobin-receptor gene and tonB-exbB-exbD operon in E. coli (Stojiljkovic \& Srinivasan, 1997). In analogous experiments, we attempted to reconstitute enterobactin utilization in an E. coli ton $B$ mutant and in E. coli fepA mutants. We introduced the Bordetella ton $B-e x b B-e x b D$ operon and $f e p A$ homologue, $b f e A$, into these mutants on compatible plasmids; however, we were only able to demonstrate apparently very inefficient reconstitution in one fepA mutant. Among possible explanations for this result are that the level of $b f e A$ and/or ton $B-e x b B-e x b D$ expression is inadequate in these strains, or that these proteins are unstable in $E$. coli. In previous experiments where $b f e A$ was expressed in E. coli from the powerful T7 promoter and radioactively labelled, the level of labelled BfeA was very low, indicating either poor translation or perhaps degradation of BfeA (Beall \& Sanden, 1995b). Another possible explanation is the formation of inactive hybrid TonB-ExbB-ExbD complexes.

The results shown in this work further suggest the presence of several different TonB-dependent receptors in B. bronchiseptica and B. pertussis, since tonB mutants of these species were unable to utilize various iron complexes (Table 2). These receptors include the ferric enterobactin receptor (BfeA), and unidentified receptors for ferric complexes of alcaligin, ferrichrome and desferrioxamine B. Additionally, there must be at least one TonB-dependent receptor for haem sources, since utilization of haemin and haemoglobin was TonB dependent in B. bronchiseptica and B. pertussis (Table $2)$. Together with the iron-regulated BfrA, BfrB and $\mathrm{BfrC}$ proteins that have unknown ligand specificity, this brings the minimal number of predicted TonB-dependent outer-membrane receptors in this genus to eight. Since the only known siderophore produced by
Bordetella species is alcaligin (Moore et al., 1995; Brickman \& Armstrong, 1996a), the biological significance of the complex array of exogenous iron complex utilization systems present in the genus Bordetella is as yet unknown.

\section{ACKNOWLEDGEMENTS}

M.L.N. was supported by an American Society for Microbiology/National Centers for Infectious Disease Postdoctoral fellowship. We are grateful to Igor Stojiljkovic for albomycin. We thank Tim Brickman and Rob Weyant for Bordetella and colicin-overproducing strains (Brickman \& Armstrong, 1996b), and Holly Starling for excellent technical support.

\section{REFERENCES}

Ahmer, B. M. M., Thomas, M. G., Larson, R. A. \& Postle, K. (1995). Characterization of the exbBD operon of Escherichia coli and the role of ExbB and ExbD in TonB function and stability. J Bacteriol 177, 4742-4747.

Anton, M. \& Heller, K. J. (1991). Functional analysis of a Cterminally altered TonB protein of Escherichia coli. Gene 105, 23-29.

Beall, B. (1998). Two iron-regulated putative ferric siderophore receptor genes in Bordetella bronchoseptica and Bordetella pertussis. Res Microbiol 149, 189-201.

Beall, B. \& Hoenes, T. (1997). An iron-regulated outer-membrane protein specific to Bordetella bronchiseptica and homologous to ferric siderophore receptors. Microbiology 143, 135-145.

Beall, B. \& Sanden, G. N. (1995a). Cloning and initial characterization of the Bordetella pertussis fur gene. Curr Microbiol 30, $1-4$.

Beall, B. \& Sanden, G. N. (1995b). A Bordetella pertussis fepA homologue required for utilization of exogenous ferric enterobactin. Microbiology 141, 3193-3205.

Bitter, W., Tommassen, J. \& Weisbeek, P. J. (1993). Identification and characterization of the exbB, exbD and ton $B$ genes of Pseudomonas putida WCS358: their involvement in ferric pseudobactin transport. Mol Microbiol 7, 117-130.

Braun, V. (1995). Energy-coupled transport and signal transduction through the Gram-negative outer membrane via TonBExbB-ExbD-dependent receptor proteins. FEMS Microbiol Rev 16, 295-307.

Braun, V., Gaisser, S., Hermann, C., Kampfenkel, K., Killmann, H. \& Traub, I. (1996). Energy-coupled transport across the outer membrane of Escherichia coli: ExbB binds ExbD and TonB in vitro, and leucine 132 in the periplasmic region and aspartate 25 in the transmembrane region are important for ExbD activity. $J$ Bacteriol 178, 2836-2845.

Brickman, T. J. \& Armstrong, S. K. (1995). Bordetella pertussis fur gene restores iron repressibility of siderophore and protein expression to deregulated Bordetella bronchiseptica fur mutants. J Bacteriol 177, 268-270.

Brickman, T. J. \& Armstrong, S. K. (1996a). Purification, spectroscopic analysis and biological activity of the macrocyclic dihydroxamate siderophore alcaligin produced by Bordetella bronchiseptica. Biometals 9, 191-203.

Brickman, T. J. \& Armstrong, S. K. (1996b). Colicins B and 1a as novel counterselective agents in interspecies conjugal DNA transfers from colicin-sensitive Escherichia coli donors to other gram-negative recipient species. Gene 178, 39-42. 
Brown, D. R. \& Parker C. D. (1987). Cloning of the filamentous hemagglutinin of Bordetella pertussis and its expression in Escherichia coli. Infect Immun 55, 154-161.

Calderwood, S. B. \& Mekalanos, J. J. (1987). Iron regulation of Shiga-like toxin expression in Escherichia coli is mediated by the fur locus. J Bacteriol 169, 4759-4764.

Evans, J. S., Levine, B. A., Trayer, I. P., Dorman, C. J. \& Higgins, C. F. (1986). Sequence-imposed stuctural constraints in the TonB protein of E. coli. FEBS Microbiol Lett 208, 211-216.

Fischer, E., Gunter, K. \& Braun, V. (1989b). Involvement of ExbB and TonB in transport across outer membrane of Escherichia coli: phenotypic complementation of exb mutants by overexpressed tonB and physical stabilization of TonB and ExbB. $J$ Bacteriol 171, 5127-5134.

Gudmundsdottir, A., Bell, P. E., Lundrigan, M. D., Bradbeer, C. \& Kadner, R. J. (1989). Point mutations in a conserved region (TonB box) of Escherichia coli outer membrane protein BtuB affect vitamin $\mathrm{B}_{12}$ transport. J Bacteriol 171, 6526-6533.

Hannavy, K., Barr, G. C., Dorman, C. J. \& 7 other authors (1990). TonB protein of Salmonella typhimurium, a model for signal transduction between membranes. J Mol Biol 216, 897-910.

Hantke, K. (1990). Dihydroxylbenzolyserine - a siderophore for E. coli. FEMS Microbiol Lett 67, 5-8.

Hawley, D. K. \& McClure, W. R. (1983). Compilation and analysis of Escherichia coli promoter DNA sequences. Nucleic Acids Res 11, 2237-2255.

Heller, K. J., Kadner, R. J. \& Günter, K. (1988). Suppression of the btuB451 mutation by mutations in the ton $B$ gene suggests a direct interaction between TonB and TonB-dependent receptor proteins in the outer membrane of Escherichia coli. Gene 64, 147-153.

Henderson, D. P. \& Payne, S. M. (1994). Vibrio cholerae transport systems : roles of heme and siderophore iron transport in virulence and identification of a gene associated with multiple iron transport systems. Infect Immun 62, 5120-5125.

Higgins, N. P. \& Hillyard, D. (1988). Primary structure and mapping of the hupA gene of Salmonella typhimurium. J Bacteriol 170, 5751-5758.

Jarosik, G. P., Sanders, J. D., Cope, L. D., Muller-Eberhard, U. \& Hansen, E. J. (1994). A functional tonB gene is required for both utilization of heme and virulence expression by Haemophilus influenzae type b. Infect Immun 62, 2470-2477.

Jaskula, J. C., Letain, T. E., Roof, S. K., Skare, J. T. \& Postle, K. (1994). Role of the TonB amino terminus in energy transduction between membranes. J Bacteriol 176, 2325-2338.

Kadner, R. J. (1990). Vitamin B12 transport in Escherichia coli: energy coupling between membranes. Mol Microbiol 4, 2027-2033.

Kampfenkel, K. \& Braun, V. (1992). Membrane topology of the Escherichia coli ExbD protein. J Bacteriol 174, 5485-5487.

Kampfenkel, K. \& Braun, V. (1993). Topology of the ExbB protein in the cytoplasmic membrane of Escherichia coli. J Biol Chem 268, 6050-6057.

Karlsson, M., Hannavy, K. \& Higgins, C. F. (1993). A sequencespecific function for the $\mathrm{N}$-terminal signal-like sequence of the TonB protein. Mol Microbiol 8, 379-388.

Keen, N. T., Kobayashi, D. \& Trollinger, D. (1988). Improved broad-host range plasmids for DNA cloning in Gram-negative bacteria. Gene 70, 191-197.
Klebba, P. E., Rutz, J. M., Liu, J. \& Murphy, C. K. (1993). Mechanisms of TonB-catalyzed iron transport through the enteric bacterial cell envelope. J Bioenerg Biomembr 25, 603-611.

Koebnik, R. (1993). The molecular interaction between components of TonB-ExbBD-dependent and of the TolQRA-dependent bacterial uptake mechanisms. Mol Microbiol 9, 219.

von Koenig, C. H. W., Tacken, A. \& Finger, H. (1988). Use of supplemented Stainer-Scholte broth for the isolation of Bordetella pertussis from clinical material. J Clin Microbiol 26, 2558- 2560.

Larsen, R. A., Wood, G. E. \& Postle, K. (1993). The conserved proline-rich motif is not essential for energy transduction by Escherichia coli TonB protein. Mol Microbiol 10, 943-953.

Larsen, R. A., Thomas, M. G., Wood, G. E. \& Postle, K. (1994). Partial suppression of an Escherichia coli TonB transmembrane domain mutation (delV17) by a missense mutation in ExbB. Mol Microbiol 13, 627-640.

Moore, C. H., Foster, L.-A., Gerbig, D. G., Jr, Dyer, D. W. \& Gibson, B. W. (1995). Identification of alcaligin as the siderophore produced by Bordetella pertussis and Bordetella bronchiseptica. J Bacteriol 177, 1116-1118.

Poole, K., Zhao, Q., Neshat, S., Heinrichs, D. E. \& Dean, C. R. (1996). The Pseudomonas aeruginosa tonB gene encodes a novel TonB protein. Microbiology 142, 1449-1458.

Porra, R. J., Langman, L., Young, L. G. \& Gibson, F. (1972). The role of ferric enterochelin esterase in enterochelin-mediated iron transport and ferrochelatase activity in Escherichia coli. Arch Biochem Biophys 153, 74-78.

Postle, K. (1993). TonB protein and energy transduction between membranes. J Bioenerg Biomembr 25, 591-601.

Skare, J. T. \& Postle, K. (1991). Evidence for a TonB-dependent energy transduction complex in Escherichia coli. Mol Microbiol 5, 2883-2890.

Skare, J. T., Ahmer, M. M., Seachord, C. L., Darveau, R. P. \& Postle, K. (1993). Energy transduction between membranes: TonB, a cytoplasmic membrane protein, can be chemically crosslinked in vivo to the outer membrane receptor FepA. J Biol Chem 268, 16302-16308.

Stibitz, S. (1994). Use of conditionally counterselective suicide vectors for allelic exchange. Methods Enzymol 235, 458-465.

Stojiljkovic, I. \& Srinivasan, N. (1997). Neisseria meningitidis ton $B$, exbB, and exbD genes: Ton-dependent utilization of protein-bound iron in Neisseriae. J Bacteriol 179, 805-812.

Traub, I., Gaiser, S. \& Braun, V. (1993). Activity domains of the TonB protein. Mol Microbiol 8, 409-423.

Weiss, A. A. \& Hewlett, E. L. (1986). Virulence factors of Bordetella pertussis. Annu Rev Microbiol 40, 661-686.

Wiggerich, H., Klauke, B., Köplin, R., Priefer, U. B. \& Pühler, A. (1997). Unusual structure of the tonB-exb DNA region of Xanthomonas campestris pv. campestris: ton $B, \operatorname{exbB}$, and exbD1 are essential for ferric iron uptake, but exbD2 is not. J Bacteriol 179, 7103-7110.

Zhao, Q., Li, X., Mistry, A., Srikumar, R., Zhang, L., Lomovskaya, O. \& Poole, K. (1998). Influence of the TonB energy-coupling protein on efflux-mediated multidrug resistance in Pseudomonas aeruginosa. Antimicrob Agents Chemother 42, 2225-2231.

Received 19 January 1999; revised 16 April 1999; accepted 4 May 1999. 